\title{
Prospective multi-center study of oncologic outcomes of robot-assisted partial nephrectomy for pT1 renal cell carcinoma
}

Rachel L Kyllo ${ }^{1}$, Youssef S Tanagho ${ }^{1}$, Jihad H Kaouk ${ }^{2}$, Michael D Stifelman ${ }^{3}$, Craig G Rogers ${ }^{4}$, Shahab P Hillyer ${ }^{2}$, Shyam S Sukumar ${ }^{4}$, Kenneth G Nepple ${ }^{1}$ and Sam B Bhayani ${ }^{1,5^{*}}$

\begin{abstract}
Background: Partial nephrectomy has been increasingly recommended over radical nephrectomy for the management of small renal masses based on improved renal functional outcomes without sacrifice of oncologic effectiveness. Robot-assisted partial nephrectomy (RAPN) has been introduced in an effort to offer another minimally invasive option for nephron-sparing surgery. However, reports of RAPN have been limited to short-term perioperative outcomes. The goal of this study is to report and evaluate the initial oncologic outcomes of RAPN. Utilizing prospectively obtained data on RAPN performed by four surgeons at four separate tertiary care centers, we selected patients with unilateral, localized, non-familial, pathologically-confirmed pT1 renal cell carcinoma and a minimum postoperative follow-up of 12 months.
\end{abstract}

Methods: Utilizing prospectively obtained data on RAPN performed by four surgeons at four separate tertiary care centers, we selected patients with unilateral, localized, non-familial, pathologically-confirmed pT1 renal cell carcinoma and a minimum post-operative follow-up of 12 months. Survival analysis (disease-free, cancer-specific, and overall survival) was performed, and Kaplan-Meier curves were generated.

Results: RAPN was performed in 124 patients with a median tumor size of $3.0 \mathrm{~cm}$ (IQR 2.2-4.2 cm). Median follow-up was 29 months (range 12-46 months). Positive parenchymal surgical margins occurred in two patients (1.6\%), both of whom were recurrence-free at 30 and 34 months after surgery. The three-year Kaplan-Meier estimated disease-free survival was $94.9 \%$, cancer-specific survival was $99.1 \%$, and overall survival was $97.3 \%$.

Conclusions: In our cohort of patients with small renal carcinomas who were followed for a median of 29 months, recurrence and survival outcomes were similar to those reported for open and laparoscopic partial nephrectomy. Further long-term outcomes will be needed to definitively claim that RAPN is oncologically equivalent to other surgical approaches.

Keywords: Renal cell carcinoma, Partial nephrectomy, Robotic partial nephrectomy, Oncologic outcomes, Nephron-sparing surgery

\footnotetext{
* Correspondence: bhayani@wustl.edu

'Division of Urology, Washington University School of Medicine, St Louis, MO, USA

${ }^{5}$ Division of Urology, Washington University School of Medicine, 660 S. Euclid Avenue, St. Louis, MO 63110, USA

Full list of author information is available at the end of the article
}

\section{Biomed Central}

(c) 2012 Kyllo et al.; licensee BioMed Central Ltd. This is an Open Access article distributed under the terms of the Creative Commons Attribution License (http://creativecommons.org/licenses/by/2.0), which permits unrestricted use, distribution, and reproduction in any medium, provided the original work is properly cited. 


\section{Background}

The incidence of small renal neoplasia is increasing, likely secondary to the increasing use of cross-sectional imaging [1]. Although, traditionally, radical nephrectomy has been performed for the definitive treatment of suspected small renal cell cancers, partial nephrectomy has become increasingly popular as a nephron-sparing option [2]. The American Urological Association's guidelines for management of the clinical T1 renal mass place partial nephrectomy as a standard treatment option [3].

Partial nephrectomy may be accomplished via a variety of techniques. Open partial nephrectomy (OPN) has been shown to have excellent long-term oncologic outcomes which are similar to radical nephrectomy [4,5]. Laparoscopic partial nephrectomy (LPN) was developed as a minimally invasive alternative to open partial nephrectomy, and studies have also shown cancer control comparable to OPN $[6,7]$. More recently, robot-assisted partial nephrectomy (RAPN) has been developed as another option for the excision of a small renal mass. Preliminary studies have shown an acceptable learning curve with feasible results [8-11]. Complications of the RAPN approach are appropriate $(15.8 \%$ overall complication rate) [12], as are initial perioperative pathological outcomes (positive margin rate of $1.6 \%$ - 4.1\%) $[8,13-$ $15]$. We previously reported multi-institutional analyses of perioperative and functional outcomes of RAPN- including complication rates, blood loss, warm ischemia time, post-operative changes in glomerular filtration rate, and hospital stay- and demonstrated favorable outcomes in all of these parameters $[8,15]$.

Despite the favorable perioperative results of RAPN, there are no current studies focused on survival outcomes of this newer procedure. Nascent procedures should undergo analysis and reporting of oncologic outcomes to ensure that survival curves are not inferior to those of established

Table 1 Demographic and pre-operative patient characteristics in $\mathbf{1 2 4}$ patients undergoing RAPN

\begin{tabular}{ll}
\hline No. Patients & $\mathbf{1 2 4}$ \\
\hline Median Age (IQR) years & $58.0(52.6-67.6)$ \\
Median months follow-up (IQR) & $29.0(25.1-34.0)$ \\
No. Sex (\%) & \\
Male & $80(64.5)$ \\
Female & $44(35.5)$ \\
No. Race (\%) & \\
Caucasian & $100(80.6)$ \\
African American & $14(11.3)$ \\
Other & $10(8.1)$ \\
Median ASA classification (IQR) & $2(2-3)$ \\
Median Charlson Comorbidity Index (IQR) & $1(0-2)$ \\
Median BMI (IQR) kg/m² & $28.3(25.1-33.6)$ \\
\hline
\end{tabular}

procedures. Previous reports of OPN and LPN show disease-free survival (DFS) rates of 83\% - 98\%, cancer-specific survival (CSS) rates of $82 \%$ - 99\%, and overall survival (OS) rates of $72 \%-96 \%$, respectively [4-6,16-20]. A formal assessment of oncologic outcomes of RAPN is absolutely necessary to demonstrate comparative efficacy of the robotic approach. Therefore, the primary goal of this multi-center study is to examine the intermediate-term oncologic outcomes of pT1 renal cell cancers which have been treated with RAPN. The goal will be to assess an "oncologic checkpoint" to ensure that the outcomes of this procedure are not oncologically inferior to the excellent outcomes that have been reported with LPN and OPN.

\section{Results}

Demographic and clinical data from the study group $(\mathrm{n}=124)$ are presented in Table 1 . Median patient age was 58 years, and median age-adjusted Charlson Comorbidity Index was 1 . Table 2 demonstrates the pathological characteristics of the tumors and data on cancer outcomes. Median tumor size was $3.0 \mathrm{~cm}$, and 107 (86.3\%) were staged pT1a, with the remaining $17(13.7 \%)$ staged as pT1b. Twelve (9.7\%) tumors were classified as Fuhrman grade I, 65 (52.4\%) as Fuhrman grade II, 30 (24.2\%) as Fuhrman grade III, and one $(0.8 \%)$ as Fuhrman grade IV; grade was not reported in 16 patients. The predominant RCC subtype was clear cell

Table 2 Tumor characteristics and oncologic outcomes in 124 patients undergoing RAPN

\begin{tabular}{ll}
\hline Median cm Pathological Size (IQR) & $\mathbf{3 . 0}(\mathbf{2 . 2 - 4 . 2 )}$ \\
\hline No. Side (\%) & $63(50.8)$ \\
Left & $61(49.2)$ \\
Right & \\
No. Histology (\%) & $83(66.9)$ \\
Clear & $26(21.0)$ \\
Papillary & $12(9.7)$ \\
Chromophobe & $3(2.4)$ \\
Other & \\
No. Fuhrman Grade (\%) & $12(9.7)$ \\
I & $65(52.4)$ \\
II & $30(24.2)$ \\
III & $1(0.8)$ \\
IV & $16(12.9)$ \\
Unavailable & \\
No. Pathological Stage (\%) & $107(86.3)$ \\
T1a & $17(13.7)$ \\
T1b & $2(1.6)$ \\
No. Positive Margins (\%) & $1(0.8)$ - ipsilateral \\
No. Renal Recurrences (\%) & $1(0.8)$ \\
No. Distant Recurrences (\%) &
\end{tabular}


(66.9\%), with a smaller proportion of papillary (21.0\%) and chromophobe $(9.7 \%)$ subtypes. Positive parenchymal margins occurred in two cases (1.6\%); both of these patients had pT1a RCC and were free of recurrence at 30 and 34 months of follow-up.

Median follow-up was 29.0 months (IQR 25.1-34.0 months, total range $12-46$ months). Two patients (1.6\%) had recurrence of renal cell cancer at 16 and 35 months post-RAPN (details described below). One of the recurrences was in the ipsilateral kidney, and the other had widespread intraperitoneal metastases. Both patients with recurrence had negative surgical margins on their original RAPN specimens.

The one ipsilateral recurrence occurred in a patient with pT1a, Fuhrman grade II clear RCC. A completely endophytic $1.7 \mathrm{~cm}$ hypervascular renal lesion developed 35 months after $\mathrm{RPN}$; this recurrence was treated with percutaneous cryoablation. No diagnostic biopsy of the recurrence was obtained, but this event is coded as a recurrence for the purposes of this communication. The second recurrence was a presumed metastatic renal cell cancer. This patient originally had a $2.7 \mathrm{~cm}$ pT1a, Fuhrman grade II papillary RCC excised with negative margins. The patient developed metastases (nodal, solid organ, and omental studding) 16 months after RAPN. Tissue diagnosis of the metastases was not definitive, but renal cell cancer was the only confirmed malignancy in this patient. This patient expired seven months after metastasis development (23 months after the original RAPN) and is coded as a cancer-specific mortality for this study, although an alternative secondary malignancy cannot be ruled out. There was no evidence of tumor spillage, seeding, or known divergence from oncologic standards during the patient's original RAPN procedure, and explanation of the pathophysiology of this recurrence is not possible.

Death from other non-cancer causes occurred in three individuals. One patient experienced a cardiovascular-related morbidity 24 months after surgery. A second patient expired from metastatic breast cancer 43 months after RAPN. The last morbidity was a death from an unknown cause 22 months after surgery; however, this patient was disease-free on imaging performed prior to expiration.

Kaplan-Meier curves were generated for 124 patients with pT1 RCC. The three-year Kaplan-Meier estimated diseasefree survival [Figure 1] rate was 94.9\% (95\% CI: 86.5\% $100 \%)$. The three-year cancer-specific survival [Figure 2] was estimated at 99.1\% (95\% CI: $97.3-100 \%)$, and the overall survival rate was estimated at $97.3 \%$ (95\% CI: $94.3 \%$ 100\%) [Figure 3].

\section{Discussion}

Nephron-sparing surgery (NSS) has emerged as a standard of care for treating localized renal malignancy [3]. Numerous studies have demonstrated that NSS is a safe and effective alternative to radical nephrectomy for patients with small renal tumors, and the use of NSS has been associated with improved renal functional outcomes and significant decreases in cardiovascular disease and overall mortality [21,22].

Open partial nephrectomy is a well-established procedure with studies showing similar oncologic outcomes to radical nephrectomy. Laparoscopic partial nephrectomy has also been studied with similarly satisfactory oncologic outcomes. Robot-assisted partial nephrectomy has not

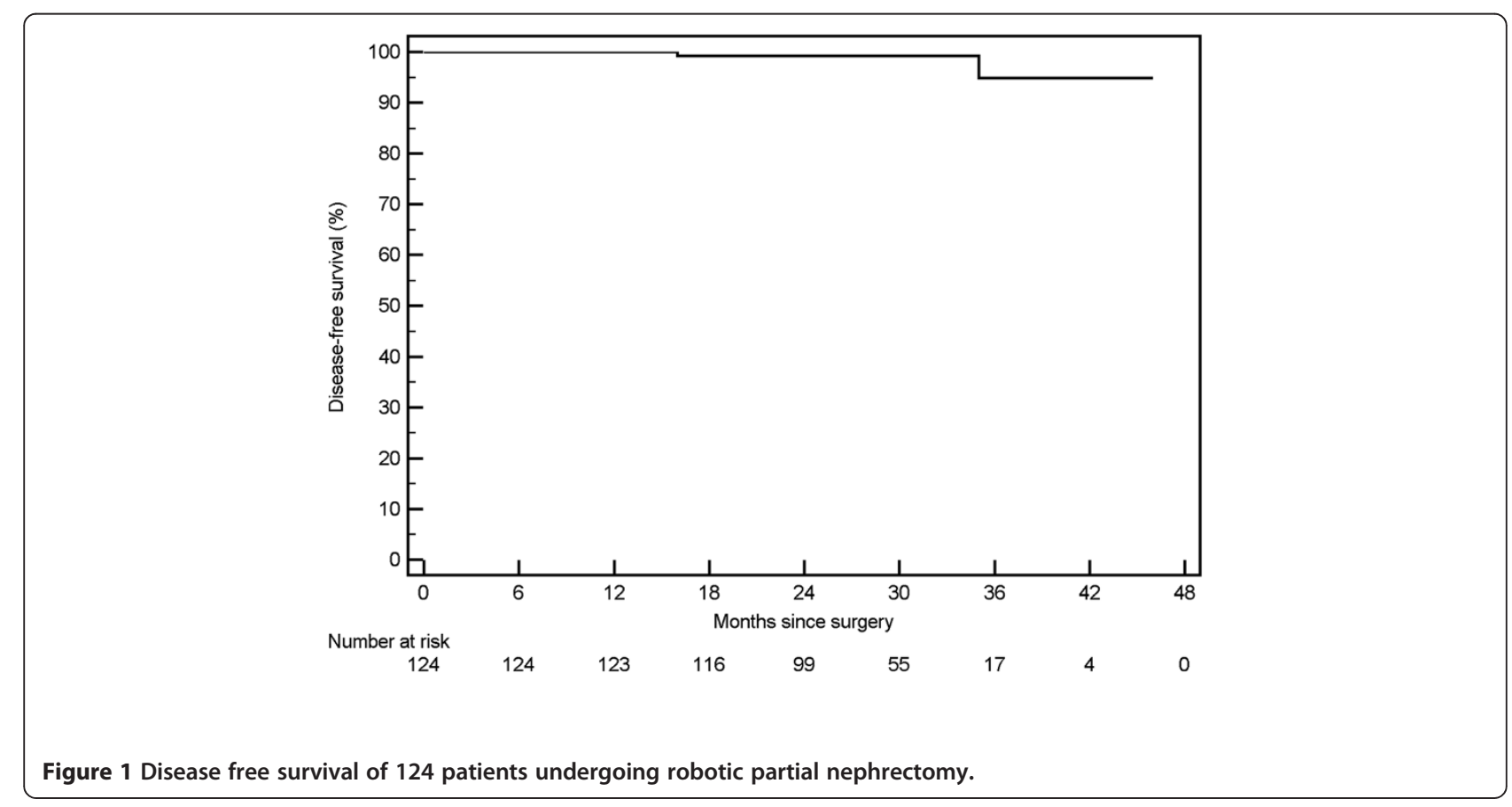




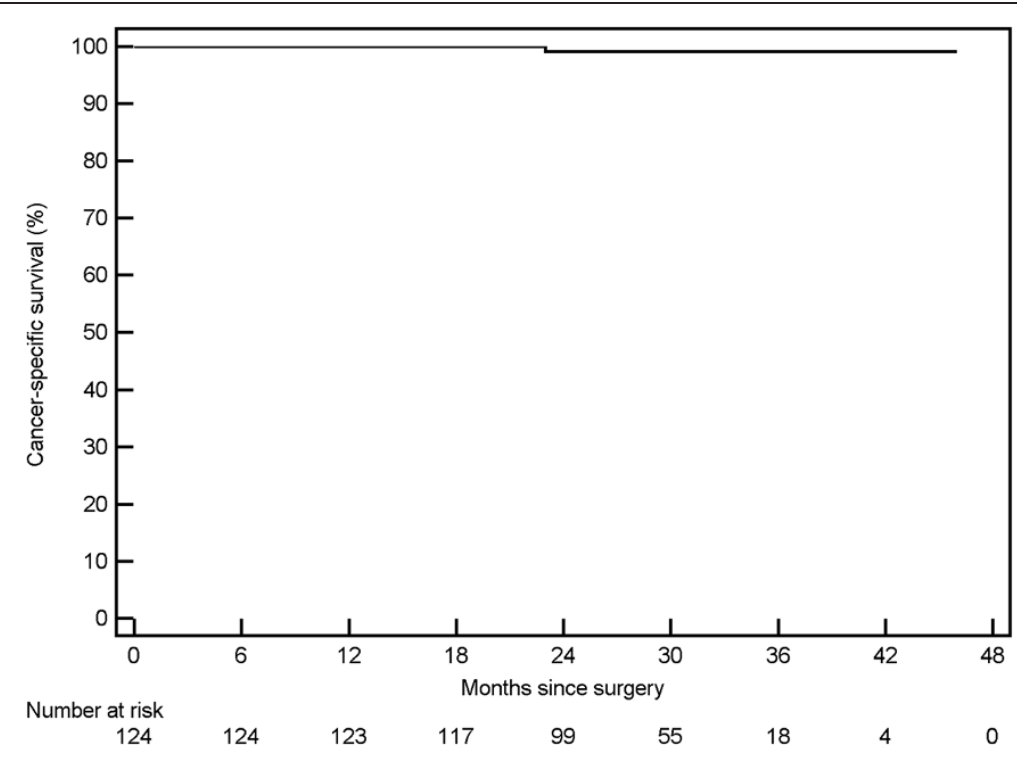

Figure 2 Cancer-specific survival of 124 patients undergoing robotic partial nephrectomy.

been studied with regards to intermediate-term survival outcomes, as the procedure was first reported in 2004 and did not appear in increasing numbers in the literature until 2008 [23]. As such, the existing literature on RAPN has focused on feasibility, perioperative outcomes, and surgical margin status. As with all new oncologic procedures, it is critical to report early outcomes to ensure that survival curves are appropriate relative to established procedures. In this multi-center study with a median follow-up of 2.5 years and a maximum follow-up of nearly four years, we report intermediate-term oncologic outcomes similar to those that have been previously published for LPN and OPN.

Table 3 outlines survival outcomes of OPN and LPN from several recent studies reported in the literature. The threeyear DFS herein for pT1 ( $a$ and $b$ ) lesions $(94.9 \%$ ) is similar

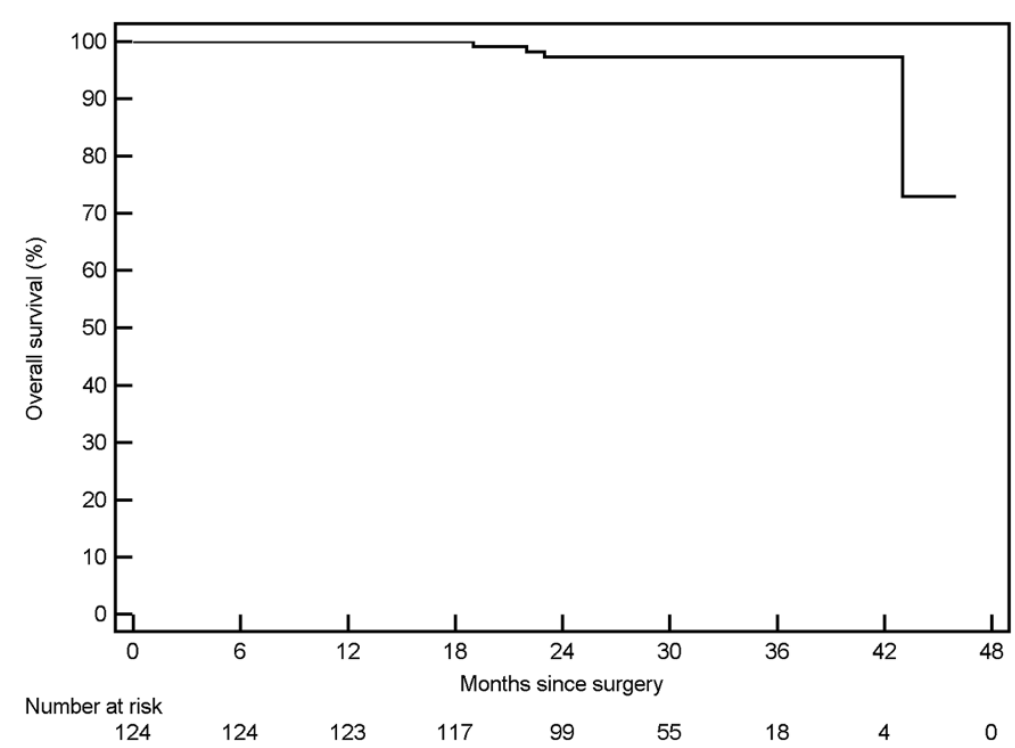

Figure 3 Overall survival of 124 patients undergoing robotic partial nephrectomy. 
Table 3 Reported oncologic outcomes of open (OPN) and laparoscopic partial nephrectomy (LPN).MFS = metastasis-free survival; DFS = disease-free survival; CSS = cancer-specific survival; OS = overall survival

\begin{tabular}{|c|c|c|c|c|c|c|c|}
\hline Study Name & Stages & $N$ & Surgery & $\begin{array}{l}\text { Follow-up } \\
\text { (months) }\end{array}$ & MFS or DFS & CSS & OS \\
\hline Lerner et al. $1996^{15}$ & $\begin{array}{l}\text { Robson } \\
\text { stage } \leq \|\end{array}$ & 185 & OPN & 44.4 (mean) & 83 \% MFS @ 5y & $89 \% @ 5 y$ & $77 \% @ 5 y$ \\
\hline Hafez et al. $1999^{5}$ & $\begin{array}{l}\text { T1, T2, } \\
\text { T3a, T3b }\end{array}$ & 485 & OPN & 47 (mean) & NM & 82 \%-99 \% @ 5y & $81 \% @ 5 y$ \\
\hline $\begin{array}{l}\text { Belldegrun et al. } \\
1999^{16}\end{array}$ & All & 146 & OPN & 74 (median) & NM & $98 \%$ & $86 \%$ \\
\hline Lau et al. $2000^{4}$ & All & 164 & OPN & 40.8 (median) & 98 \% MFS @ 5y & $98 \% @ 5 y$ & $91 \% @ 5 y$ \\
\hline \multirow{2}{*}{$\begin{array}{l}\text { Permpongkosol } \\
\text { et al. } 2006{ }^{17}\end{array}$} & $\mathrm{~T} 1$ & 85 & LPN & 40.4 (mean) & 91.4 \% DFS @ 5y & NM & $93.75 \%$ @ $5 y$ \\
\hline & $\mathrm{T} 1$ & 58 & OPN & 49.7 (mean) & 97.6\% DFS @ 5y & NM & 95.8 \% @ 5y \\
\hline \multirow[t]{2}{*}{ Gill et al. $2007^{6}$} & $\mathrm{~T} 1$ & 514 & LPN & 14.4 (median) & NM & 99.3 \% @ 3y & NM \\
\hline & $\mathrm{T} 1$ & 676 & OPN & 33.6 (median) & NM & $99.2 \% @ 3 y$ & NM \\
\hline Joniau et al. $2009^{18}$ & $\mathrm{~T} 1 \mathrm{~b}$ & 67 & OPN & 40.2 (median) & 84 \% DFS @ 5y & $99 \%$ @ @ y & $72 \% @ 5 y$ \\
\hline \multirow[t]{2}{*}{ Lane et al. $2010^{19}$} & All & 499 & LPN & 48 (median) & 86-97 \% MFS @ 5y & 93-97 \% @ 5y & NM \\
\hline & All & 762 & OPN & 68.4 (median) & 97 \% MFS @ 5y & 97 \%-99 \% @ 5y & NM \\
\hline Present study & $\mathrm{T} 1$ & 124 & RAPN & 29 (median) & 94.9 \% DFS @ 3y & $99.1 \%$ @ 3y & $97.3 \%$ @ 3y \\
\hline
\end{tabular}

to those previously reported for OPN and LPN (84\% 97.6\%). Other papers have not reported DFS, but instead documented metastasis-free survival at $83 \%-98 \%$. RAPN in this study shows a three-year Kaplan-Meier estimated CSS of $99.1 \%$ at a median follow-up of 29 months. This compares to other studies of OPN and LPN, which have reported CSS at three years or greater of $82 \%-99 \%$ and 93\% - 99.3\%, respectively. Additionally, the three-year OS herein $(97.3 \%)$ is comparable to those demonstrated in the literature for OPN and LPN (Table 3).

The patterns of recurrence are not known yet for robotic partial nephrectomy. For open partial nephrectomy, the most common sites of metastasis include lymph nodes, lungs, contralateral kidney, bone, brain, and liver. In this series, there was one case of peritoneal implantation, but without pathological confirmation that this was renal cell, it is hard to make any firm conclusions. Nevertheless, we have included it as a cancer related mortality for the purposes of reporting. It will be interesting to monitor sites of recurrence in the future.

Thus, the RAPN results reported here appear to be generally comparable to those previously documented for OPN and LPN. Longer term follow-up will be necessary to ensure no divergence from the standards set by OPN and LPN. It is critical to note that a detailed statistical comparison of outcomes between series is not possible secondary to differences among studies in follow-up, pathology, tumor grades, RCC subtypes, and reported data. Additionally, since the majority of cases are less than $4 \mathrm{~cm}$ in this study, perhaps even active surveillance would have resulted in similar outcomes. Again, long term studies are needed.
Despite these reasonable outcomes, caution is advised in interpreting the results of this study. As the outcomes are still at less than four-year follow-up, broad conclusions cannot be made regarding long-term oncologic outcomes. Moreover, studies have shown that the risk of RCC recurrence is highest between three and five years post-surgery $[24,25]$. Thus, more follow-up will be necessary to further evaluate the long-term oncologic outcomes of RAPN. Secondly, the surgeons herein are all high-volume robotic renal surgeons practicing in tertiary-care academic medical centers, and the generalization of these results to lower volume surgeons is unknown. Prior reports have identified improved outcomes comparing high-volume to low-volume surgeons in some procedures [26,27]. Nevertheless, this same argument can be applied to most of the OPN and LPN outcomes literature. Another concern is that this study is a retrospective review of prospectively maintained databases, so there are likely inherent unrecognized selection biases that would not exist in a randomized study. Lastly, it is important for surgeons to consider that oncologic outcomes are only one factor in choosing a treatment modality; other issues such as complications, ease of performance, patient acceptance, and cost are important issues which can influence treatment decisions. There certainly is no detectable superiority of RAPN from a cancer control perspective. Nevertheless, RAPN appears to have appropriate survival outcomes in this study. Longer term follow-up is needed to ensure no divergence in survival from established standards. Additionally, caution is advised in the interpretation of any survival analysis, since a recent randomized study showed a slightly lower overall survival 
with partial nephrectomy compared to radical nephrectomy [28].

\section{Conclusions}

This study adds to the literature by establishing the first multicenter evaluation of oncologic outcomes following RAPN. In patients with localized renal cell cancer measuring less than $4 \mathrm{~cm}$ (and perhaps $7 \mathrm{~cm}$, but overall numbers are low), RAPN was found to have a low rate of positive surgical margins and disease-free, cancer-specific, and overall survival rates similar to those previously reported for other surgical approaches. Although this "oncologic checkpoint" for RAPN is promising on a preliminary basis, further follow-up will be needed to critically assess the long-term oncologic outcomes of RAPN.

\section{Methods}

Prospective data were collected from databases approved by institutional review boards (IRB), and separate IRB approval was obtained for pooling the data for a multi-center study. Demographic, clinical, and follow-up data were collected prospectively on patients who underwent RAPN at four tertiarycare U.S. academic urological centers from June 2006 to June 2009. All patients who underwent RAPN for a pathologically confirmed pT1 renal cell carcinoma (RCC) with a minimum follow-up of 12 months were included. Patients with bilateral synchronous tumors, known familial cancer syndrome, or known metastatic disease were excluded. Hence, the study cohort was limited to small $(<7 \mathrm{~cm})$, sporadic, organ-confined RCC. Comorbid conditions were assessed with the American Association of Anesthesiologists Score (ASA) and the ageadjusted Charlson Comorbidity Index [29].

RAPN was performed using previously published techniques [30-32]. The oncologic follow-up protocol consisted of physical examination and cross-sectional imaging of the chest and abdomen at three to six months postoperatively and annually thereafter to rule out recurrence. Additional imaging was left to the discretion of the primary urologist or primary care physician. Survival outcomes were evaluated using Kaplan-Meier survival analysis. Statistical analysis was completed using SPSS ${ }^{\circledR} 15$, Medcalc 11.4, and Microsoft Excel 2007.

\section{IRB approval and consent}

Washington University School of Medicine Human Studies Committee, Cleveland Clinic Institutional Review Board, Institutional Review Board, NYU Langone Medical Center, Henry Ford Institutional Review Board.

NOTE: Consent was obtained from patients for participation in this study.

\footnotetext{
Abbreviations

RCC: Renal cell carcinoma; NSS: Nephron-sparing surgery; RAPN: Robotassisted partial nephrectomy; OPN: Open partial nephrectomy; LPN: Laparoscopic partial nephrectomy; DFS: Disease-free survival; CSS: Cancer-specific survival; OS: Overall survival; MFS: Metastasis-free survival.
}

\section{Competing interests}

Rachel L. Kyllo, B.S.: No competing interests exist. Youssef S. Tanagho, M.D., M.P.H.: No competing interests exist. Jihad Kaouk, M.D.: Intuitive Surgical, Inc. Michael Stifelman, M.D.: Intuitive Surgical, Inc. Craig Rogers, M.D.: Intuitive Surgical, Inc. Shahab Hillyer, M.D.: No competing interests exist. Shyam Sukumar, M.D.: No competing interests exist. Kenneth G. Nepple, M.D.: No competing interests exist. Sam B. Bhayani, M.D.: No competing interests exist.

\section{Author details}

${ }^{1}$ Division of Urology, Washington University School of Medicine, St Louis, MO, USA. ${ }^{2}$ Glickman Urological Institute, Cleveland Clinic, Cleveland, $\mathrm{OH}$ USA. ${ }^{3}$ Department of Urology, NYU Langone School of Medicine, New York, NY, USA. ${ }^{4}$ Department of Urology, Henry Ford Hospital, Detroit, MI, USA.

${ }^{5}$ Division of Urology, Washington University School of Medicine, 660 S. Euclid Avenue, St. Louis, MO 63110, USA.

\section{Authors' contributions}

RLK: data acquisition, data analysis and interpretation, drafting of manuscript, YST: drafting of manuscript; data analysis and interpretation; critical revision of the manuscript, JK: data acquisition, critical revision of the manuscript, supervision, MS: data acquisition, critical revision of the manuscript, supervision, CR: data acquisition, critical revision of the manuscript, supervision, $\mathrm{SH}$ : data acquisition, critical revision of the manuscript, SS: data acquisition, critical revision of the manuscript, KGN: critical revision of the manuscript; data analysis and interpretation, SBB: conception and design, drafting of manuscript, data acquisition, critical revision of the manuscript, supervision. All authors read and approved the final manuscript.

Received: 13 December 2011 Accepted: 30 April 2012

Published: 30 April 2012

\section{References}

1. Sun M, Thuret $R$, Abdollah F, et al: Age-adjusted incidence, mortality, and survival rates of stage-specific renal cell carcinoma in North America: a trend analysis. Eur Urol 2011, 59:135-141.

2. Cooperberg MR, Mallin K, Kane CJ, et al: Treatment trends for stage I renal cell carcinoma. J Urol 2011, 186:394-399.

3. Campbell SC, Novick AC, Belldegrun A, et al: Guideline for management of the clinical T1 renal mass. J Urol 2009, 182:1271-1279.

4. Lau WK, Blute ML, Weaver AL, et al: Matched comparison of radical nephrectomy vs nephron-sparing surgery in patients with unilateral renal cell carcinoma and a normal contralateral kidney. Mayo Clin Proc 2000, 75:1236-1242.

5. Hafez KS, Fergany AF, Novick AC: Nephron sparing surgery for localized renal cell carcinoma: impact of tumor size on patient survival, tumor recurrence and TNM staging. J Urol 1999, 162:1930-1933.

6. Gill IS, Kavoussi LR, Lane BR, et al: Comparison of 1,800 laparoscopic and open partial nephrectomies for single renal tumors. J Urol 2007, 178:41-46.

7. Allaf ME, Bhayani SB, Rogers C, et al: Laparoscopic partial nephrectomy: evaluation of long-term oncological outcome. J Urol 2004, 172:871-873.

8. Benway BM, Bhayani SB, Rogers CG, et al: Robot-assisted partial nephrectomy: an international experience. Eur Urol 2010, 57:815-820.

9. Haber GP, White WM, Crouzet S, et al: Robotic versus laparoscopic partial nephrectomy: single-surgeon matched cohort study of 150 patients. Urology 2010, 76:754-758.

10. Deane LA, Lee HJ, Box GN, et al: Robotic versus standard laparoscopic partial/wedge nephrectomy: a comparison of intraoperative and perioperative results from a single institution. J Endourol 2008, 22:947-952

11. Mottrie A, De Naeyer $G$, Schatteman $P$, et al: Impact of the learning curve on perioperative outcomes in patients who underwent robotic partial nephrectomy for parenchymal renal tumours. Eur Urol 2010, 58:127-132.

12. Spana G, Haber GP, Dulabon LM, et al: Complications after robotic partial nephrectomy at centers of excellence: multi-institutional analysis of 450 cases. J Urol 2011, 186:417-422

13. Rogers CG, Menon M, Weise ES, et al: Robotic partial nephrectomy: a multi-institutional analysis. J Robotic Surg 2008, 2:141-143.

14. Dulabon LM, Kaouk JH, Haber GP, et al: Multi-institutional analysis of robotic partial nephrectomy for hilar versus nonhilar lesions in 446 consecutive cases. Eur Urol 2011, 59:325-330. 
15. Benway BM, Bhayani SB, Rogers CG, et al: Robot assisted partial nephrectomy versus laparoscopic partial nephrectomy for renal tumors: a multi-institutional analysis of perioperative outcomes. J Urol 2009, 182(3):866-872.

16. Lerner SE, Hawkins CA, Blute ML, et al: Disease outcome in patients with low stage renal cell carcinoma treated with nephron sparing or radical surgery. 1996. J Urol 2002; 167:884-9; discussion 889-90.

17. Belldegrun A, Tsui KH, deKernion JB, et al: Efficacy of nephron-sparing surgery for renal cell carcinoma: analysis based on the new 1997 tumor-node-metastasis staging system. J Clin Oncol 1999, 17:2868-2875.

18. Permpongkosol S, Bagga HS, Romero FR, et al: Laparoscopic versus open partial nephrectomy for the treatment of pathological T1NOMO renal cell carcinoma: a 5-year survival rate. J Urol 2006; 176:1984-8; discussion 1988-9.

19. Joniau S: Vander Eeckt K, Srirangam SJ, et al: Outcome of nephron-sparing surgery for T1b renal cell carcinoma. BJU Int 2009, 103:1344-1348.

20. Lane BR, Gill IS: 7-Year oncological outcomes after laparoscopic and open partial nephrectomy. J Urol 2010, 183:473-479.

21. Huang WC, Elkin EB, Levey AS, et al: Partial nephrectomy versus radical nephrectomy in patients with small renal tumors-is there a difference in mortality and cardiovascular outcomes? J Urol 2009; 181:55-61; discussion 61-2.

22. Go AS, Chertow GM, Fan D, et al: Chronic kidney disease and the risks of death, cardiovascular events, and hospitalization. N Engl J Med 2004, 351:1296-1305.

23. Gettman MT, Blute ML, Chow GK, et al: Robotic-assisted laparoscopic partial nephrectomy: technique and initial clinical experience with DaVinci robotic system. Urology 2004, 64:914-918.

24. Hafez KS, Novick AC, Campbell SC: Patterns of tumor recurrence and guidelines for follow-up after nephron sparing surgery for sporadic renal cell carcinoma. J Urol 1997, 157:2067-2070.

25. Montie JE: Follow-up after partial or total nephrectomy for renal cell carcinoma. Urol Clin North Am 1994, 21:589-592.

26. Birkmeyer JD, Stukel TA, Siewers AE, et al: Surgeon volume and operative mortality in the United States. N Engl J Med 2003, 349:2117-2127.

27. Vickers AJ, Savage CJ, Hruza M, et al: The surgical learning curve for laparoscopic radical prostatectomy: a retrospective cohort study. Lancet Oncol 2009, 10:475-480.

28. Van Poppel H, Da Pozzo L, Albrecht W, et al: A Prospective, Randomised EORTC Intergroup Phase 3 Study Comparing the Oncologic Outcome of Elective Nephron-Sparing Surgery and Radical Nephrectomy for Low-Stage Renal Cell Carcinoma. Eur Urol 2011, 59:543-552.

29. Hall WH, Ramachandran R, Narayan $S$, et al: An electronic application for rapidly calculating Charlson comorbidity score. BMC Cancer 2004, 4:94.

30. Benway BM, Wang AJ, Cabello JM, et al: Robotic partial nephrectomy with sliding-clip renorrhaphy: technique and outcomes. Eur Urol 2009, 55:592-599.

31. Bhayani SB: da Vinci robotic partial nephrectomy for renal cell carcinoma: an atlas of the four-arm technique. J Robotic Surg; 20071:279-285.

32. Patel MN, Bhandari M, Menon M, et al: Robotic-assisted partial nephrectomy. BJU Int 2009, 103:1296-1311.

\section{Submit your next manuscript to BioMed Central and take full advantage of:}

- Convenient online submission

- Thorough peer review

- No space constraints or color figure charges

- Immediate publication on acceptance

- Inclusion in PubMed, CAS, Scopus and Google Scholar

- Research which is freely available for redistribution 